\title{
AVALIAÇÃO DO PADRÃO DE USO DO ÁLCOOL ENTRE MORADORES DE UMA REGIÃO SOCIALMENTE VULNERÁVEL
}

\author{
| Alda Gonçalves'; Andréia de Oliveira ${ }^{2}$; Helen Gandra ${ }^{3}$; Paula Assunção ${ }^{4}$; Thaís Oliveira ${ }^{5}$; Thales da Silva ${ }^{6}$ |
}

\section{RESUMO}

CONTEXTO: O álcool, entre as drogas de abuso, é a substância psicoativa mais largamente consumida no mundo. O II Levantamento Nacional de Álcool e Drogas, feito no Brasil e publicado em 2013 aponta que a taxa de dependência e/ou abuso de álcool na população é de $6,8 \%$ sendo $10,5 \%$ homens e $3,6 \%$ mulheres.

OBJETIVO(S): Neste artigo tem-se por objetivo apresentar o padrão de consumo de álcool e as intervenções realizadas em uma comunidade socialmente vulnerável, do município de Belo Horizonte, Minas Gerais.

METODOLOGIA: Realizou-se um rastreamento do consumo de álcool entre 47 participantes, com idades superiores a 18 anos, por meio do Alcohol Use Disorders Identification Test. Foi feito um estudo transversal para a análise quantitativa dos dados. Diante do cenário pesquisado, adotou-se a pesquisa ação como uma estratégia metodológica, para fazer as intervenções a partir da classificação do padrão de consumo da população estudada. As intervenções realizadas foram norteadas pelos resultados do referido teste.

RESULTADOS: Os escores do teste foram obtidos conforme as zonas de classificação do padrão de consumo de álcool, resultando: zona I, $61,7 \%$; zona II, 6,4; zona III, $14,9 \%$ e zona IV, 17,0\%. Dentre as pessoas classificadas nas zonas III e IV $25,9 \%$ residiam com crianças e adolescentes.

CONCLUSÕES: Ressalta-se a importância da manutenção de atividades de promoção à saúde e prevenção ao uso e abuso de álcool como parte do cuidado de saúde prestado pela equipe que assiste a população estudada.

\section{PALAVRAS-CHAVE: Alcoolismo; Enfermagem em saúde pública; Promoção da saúde}

\section{RESUMEN}

"Evaluación del nivel de consumo de alcohol entre los residentes de una región con vulnerabilidad social"

CONTEXTO: El alcohol de las drogas de abuso son las drogas psicoactivas más consumidas en el mundo. Lo II Nacional de Alcohol y Drogas de Encuesta, realizada en Brasil y publicado en 2013 muestra que la tasa de dependencia y / o abuso de alcohol entre la población es de 6,8\% y $10,5 \%$ hombres y $3,6 \%$ mujeres.

OBJETIVO(S): Este artículo tiene como objetivo presentar el patrón de consumo de alcohol y las intervenciones llevadas a cabo en una comunidad socialmente vulnerables, la ciudad de Belo Horizonte, Minas Gerais.

METODOLOGÍA: Había un rastro de consumo de alcohol entre los 47 participantes, mayores de 18 años, a través del Alcohol Use Disorders Identification Test (AUDIT). Un estudio transversal se llevó a cabo para el análisis cuantitativo de los datos. En el contexto investigado, se adoptó la investigación-acción como metodología para realizar intervenciones de la clasificación del patrón de consumo de la población de estudio. Las intervenciones fueron guiados por los resultados de esa prueba. RESULTADOS: Los resultados del test se obtuvieron de acuerdo a las zonas de clasificación estándar de alcohol, lo que resulta en: zona I, el 61,7\%; la zona II, 6,4; Zona III, 14,9\% y la zona IV, 17,0\%. Entre las personas clasificadas en las zonas III y IV 25,9\% vivían con niños y adolescentes. CONCLUSIONES: Hacemos hincapié en la importancia de mantener las actividades para promover la salud y prevenir el uso y abuso de alcohol como parte de la asistencia grupo prestada por el personal de atención a la población estudiada.

\section{DESCRIPTORES: Alcoholismo; Enfermería en salud pública; Promoción de la Salud}

\begin{abstract}
"Evaluation of standard of use alcohol among residents of a region socially vulnerable"

BACKGROUND: The alcohol, between drug abuse, is the most widely consumed psychoactive substance in the world. The II National Alcohol and Drug Survey, conducted in Brazil and published in 2013 shows that the rate of addiction and / or abuse of alcohol in the population is $6.8 \%$ and $10.5 \%$ men and $3.6 \%$ women.

AIM: This paper aims to present the pattern of alcohol consumption and their interventions in a socially vulnerable community, the city of Belo Horizonte, Minas Gerais.

METHODS: We conducted a trace of alcohol consumption among 47 participants, aged over 18 years, through the Alcohol Use Disorders Identification Test. A cross-sectional study was done for the quantitative analysis of the data. Against the backdrop researched, action research was adopted as a methodology to make interventions from the classification of the study population consumption pattern.

RESULTS: The test scores were obtained depending on the zone classification of the pattern of alcohol consumption, resulting in: zone I, $61,7 \%$, zone II, 6,4\%, zone III,14,9\%, zone IV, 17,0\%. Among persons classified in zones III and IV $25,9 \%$ lived with children and adolescents.

CONCLUSIONS: It emphasizes the importance of maintaining health promotion and prevention of alcohol use and abuse as part of the health care provided by thes team that assists the population studied.
\end{abstract}

\section{KEYWORDS: Alcoholism; Health promotion; Public health nursing}

Submetido em 30-09-2014

Aceite em 03-02-2015

1 Doutora em Enfermagem Psiquiátrica; Enfermeira; Professora Associada na Universidade Federal de Minas Gerais, Escola de Enfermagem, Brasil, alda@enf.ufmg.br 2 Discente de Enfermagem; Universidade Federal de Minas Gerais, Escola de Enfermagem 30130-100 Belo Horizonte, Brasil, andreiafelipe5@gmail.com 3 Discente de Enfermagem; Universidade Federal de Minas Gerais, Escola de Enfermagem 30130-100 Belo Horizonte, Brasil, helen.gandra@hotmail.com 4 Discente de Enfermagem; Universidade Federal de Minas Gerais, Escola de Enfermagem 30130-100 Belo Horizonte, Brasil, paula.g_assuncao@hotmail.com 5 Discente de Enfermagem; Universidade Federal de Minas Gerais, Escola de Enfermagem 30130-100 Belo Horizonte, Brasil, thaismo18@gmail.com 6 Discente de Enfermagem; Universidade Federal de Minas Gerais, Escola de Enfermagem 30130-100 Belo Horizonte, Brasil, thaismo18@gmail.com

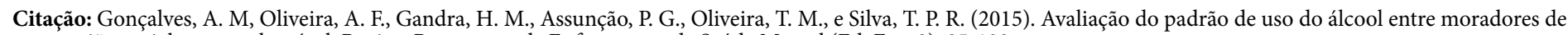
uma região socialmente vulnerável. Revista Portuguesa de Enfermagem de Saúde Mental (Ed. Esp. 2), 95-100. 


\section{INTRODUÇÃO}

As substâncias psicoativas possuem, atualmente, caráter de mercadoria, visto que estão inseridas em uma sociedade capitalista e configuram-se como mais um produto para consumo. Esse problema é complexo e exige enfoque multidisciplinar de saúde pública, pois o consumo destas substâncias ultrapassa os aspectos legais, jurídicos e sociais (Ministério da Justiça, 2013).

$\mathrm{O}$ consumo de álcool apresenta grande influência nos índices de morbimortalidade mundial, pois quando consumido em um padrão nocivo pode ocasionar comorbidades clínicas como: cirrose hepática, intoxicações, câncer hepático, entre outras doenças. Além disso, pode influenciar em acidentes, quedas e homicídios (Meloni e Laranjeira, 2004).

A maior porcentagem de pessoas que consomem álcool no Brasil concentra-se nas classes " $\mathrm{A}$ " e "B", entretanto, segundo Laranjeira, Pinsky, Zaleski,e Caetano, (2007) a classe "E" consome um maior número de doses a cada vez que se bebe. O beber excessivamente todos os dias ou a ocorrência de repetidos episódios de intoxicação pelo álcool são padrões de uso que causam prejuízos físicos, mentais e sociais para o indivíduo (Minto, Corradi-Webste, Gorayeb, Laprega, e Furtado, 2007).

O álcool, entre as drogas de abuso, é a substância psicoativa mais largamente consumida no mundo. O II Levantamento Nacional de Álcool e Drogas (II LENAD) feito no Brasil e publicado em 2013 aponta que a taxa de dependência e/ou abuso de álcool na população é de $6,8 \%$, sendo $10,5 \%$ homens e $3,6 \%$ mulheres (Laranjeira, Madruga, Ribeiro, Pinsky, Caetano,e Mitsuhiro, 2013). O perfil de consumo apresentado pelo II LENAD, anteriormente citado, é a realidade de uma comunidade socialmente vulnerável, do município de Belo Horizonte, Minas Gerais. Tendo em vista essa problemática, os profissionais da equipe de Atenção Básica responsáveis pelo atendimento de saúde dessa população, buscaram uma parceria com professores da Escola de Enfermagem da Universidade Federal de Minas Gerais para a realização de medidas de intervenção naquela realidade. Desta parceria, surgiu um projeto de pesquisa e de extensão denominado "Alcoolismo em região de risco e vulnerabilidade social: diagnóstico e intervenção".

Neste artigo, tem-se por objetivo apresentar o padrão de consumo de álcool e as intervenções realizadas em uma comunidade socialmente vulnerável do município de Belo Horizonte, Minas Gerais.

\section{METODOLOGIA}

Para a análise quantitativa dos dados utilizou-se um estudo transversal cuja amostra foi composta por 47 participantes maiores de 18 anos. Diante do cenário pesquisado, adotou-se a pesquisa ação como uma estratégia metodológica, pois permite conhecer a realidade e ao mesmo tempo fazer intervenções sobre ela. Segundo Tripp (2005), a pesquisa ação inicia-se com o reconhecimento do contexto onde o estudo é realizado, identificando as situações passíveis de intervenção, a fim de planejar uma mudança adequada.

Utilizou-se o Alcohol Use Disorders Identification Test (AUDIT) como instrumento para identificar o padrão de consumo de álcool e realizar as intervenções de acordo com os resultados obtidos. Valeu-se, também de um questionário sóciodemográfico para conhecer as seguintes variáveis: nome, idade, sexo, nível de escolaridade, número de crianças, adolescentes e adultos, por domicílio. Por meio desse questionário, identificou-se os adultos consumidores de álcool que convivem com crianças e adolescentes.

O AUDIT é constituído por 10 questões de múltipla escolha que avaliam o uso individual de álcool nos últimos 12 meses, problemas relacionados ao álcool e sintomas de dependência. As alternativas de respostas são pontuadas em ordem crescente de zero a quatro. Ao fim do teste, os pontos obtidos em cada questão são somados obtendo-se a pontuação final. Os escores finais determinam a classificação de cada participante em uma das quatro zonas, resumidas no Quadro 1 (Ministério da Justiça, 2014).

Quadro 1 - Zonas do AUDIT, pontuação e classificação

\begin{tabular}{|l|l|}
\hline Zonas do AUDIT e pontuação & Classificação \\
\hline Zona I (até 7 pontos) & $\begin{array}{l}\text { Geralmente fazem uso de } \\
\text { baixo risco de álcool ou são } \\
\text { abstêmias. }\end{array}$ \\
\hline Zona II (8 a 15 pontos) & $\begin{array}{l}\text { Usuários de risco, porém não } \\
\text { apresentam nenhum prob- } \\
\text { lema atual relacionado a esse } \\
\text { consumo. }\end{array}$ \\
\hline Zona III (16 a 19 pontos) & $\begin{array}{l}\text { Padrão de uso nocivo. São pes- } \\
\text { soas que consomem álcool e já } \\
\text { apresentam problemas decor- } \\
\text { rentes do uso de álcool. }\end{array}$ \\
\hline Zona IV (acima de 20 pontos) & $\begin{array}{l}\text { Apresentam grande chance de } \\
\text { ter um diagnóstico de de- } \\
\text { pendência de álcool. }\end{array}$ \\
\hline
\end{tabular}

Fonte: Adaptado de Ministério da Justiça (2014) 
O AUDIT foi desenvolvido pela Organização Mundial da Saúde como um método simples de investigação de uso excessivo de álcool e de ajuda na realização de intervenções (Jomar, Paixão e Abreu, 2012), publicado pela primeira vez em 1989. É considerado eficiente para a detecção do uso nocivo de álcool, devido a sua alta sensibilidade e especificidade (Magnabosco, Formigoni e Ronzani, 2007). Trata-se de um instrumento de fácil aplicação que permite resultado imediato e intervenções direcionadas a partir da zona de classificação identificada.

Foram realizadas reuniões prévias com a equipe de saúde da Atenção Básica que assiste aos moradores da região estudada, com o objetivo de planejar a entrada dos pesquisadores no cenário da pesquisa. O primeiro contato dos pesquisadores com a comunidade aconteceu em um evento no qual se comemorou o dia Mundial da Saúde. Durante esse evento, desenvolveram-se atividades educativas de promoção da saúde e iniciou-se a coleta de dados. Posteriormente deu-se continuidade à coleta dos dados em visitas domiciliares, reuniões realizadas em grupos de educação em saúde e salas de espera para atendimento médico.

Por se tratar de uma pesquisa envolvendo seres humanos, cumpriu-se as exigências formais da Resolução 466/12 do Conselho Nacional de Saúde.

\section{RESULTADOS}

Os resultados do questionário sociodemográfico e do AUDIT, permitiram gerar dados percentuais quantitativos sobre o padrão de consumo de álcool entre os sujeitos da pesquisa. Esses dados foram dispostos em tabelas de modo a possibilitar uma análise da relação entre as características sociodemográficas, o padrão de consumo de álcool e o número de crianças e adolescentes que conviviam com adultos consumidores de álcool. A Tabela 1 apresenta os principais dados sociodemográficos da amostra. Os resultados da análise de dados mostraram equilíbrio em relação ao sexo: sendo 48,9\% mulheres e $51,1 \%$ homens distribuídos em todas as faixas etárias. A maioria dos participantes, $83,0 \%$, afirmou ter como escolaridade o $1^{\circ}$ grau incompleto. Dos sujeitos da pesquisa, $65,9 \%$ convivem com crianças e adolescentes.
Tabela 1 - Dados sociodemográficos da amostra - valores expressos em porcentagem $(n=47)$ Belo Horizonte, 2013

\begin{tabular}{|l|l|l|}
\hline Sexo & Feminino & 48,9 \\
& Masculino & 51,1 \\
\hline Faixas etárias & $18-30$ anos & 14,9 \\
& $31-50$ anos & 36,2 \\
& 51 anos ou mais & 48,9 \\
\hline Escolaridade & $1^{\circ}$ Grau Incompleto & 83,0 \\
& $1^{\circ}$ Grau Completo & 10,6 \\
& $2^{\circ}$ Grau Incompleto & 2,1 \\
& $2^{\circ}$ Grau Completo & 4,3 \\
\hline Pessoas que convivem com & Sim & 65,9 \\
crianças e adolescentes & Não & 34,1 \\
\hline
\end{tabular}

Fonte: Dados da pesquisa: Alcoolismo em região de risco e vulnerabilidade social: diagnóstico e intervenção, 2013.

Observa-se que a maior parte da amostra total, $61,7 \%$, classificada na zona I, fazia uso de baixo risco ou era abstêmia. Entretanto, 21,3\% classificada na zona II e III apresentaram padrão de consumo considerado de risco ou nocivo, respectivamente, e $17,0 \%$ classificada na zona IV em níveis sugestivos de dependência.

Como demonstrado na Tabela 2, os homens foram classificados nos padrões de uso de risco ou uso nocivo, ou seja, localizaram-se nas zonas II e III, em frequência significativamente maior do que as mulheres. O mesmo foi observado em relação a zona IV que sugere possível diagnóstico de dependência de álcool. Em todas as faixas etárias houve maior concentração de pessoas com uso de baixo risco de álcool ou abstêmias. A faixa etária entre 31-50 anos apresentou 29,4\% dos participantes com uso nocivo de álcool ou propensão a dependência. Em relação a escolaridade, destaca-se que todos os classificados na zona IV afirmaram ter como escolaridade o $1^{\circ}$ grau incompleto.

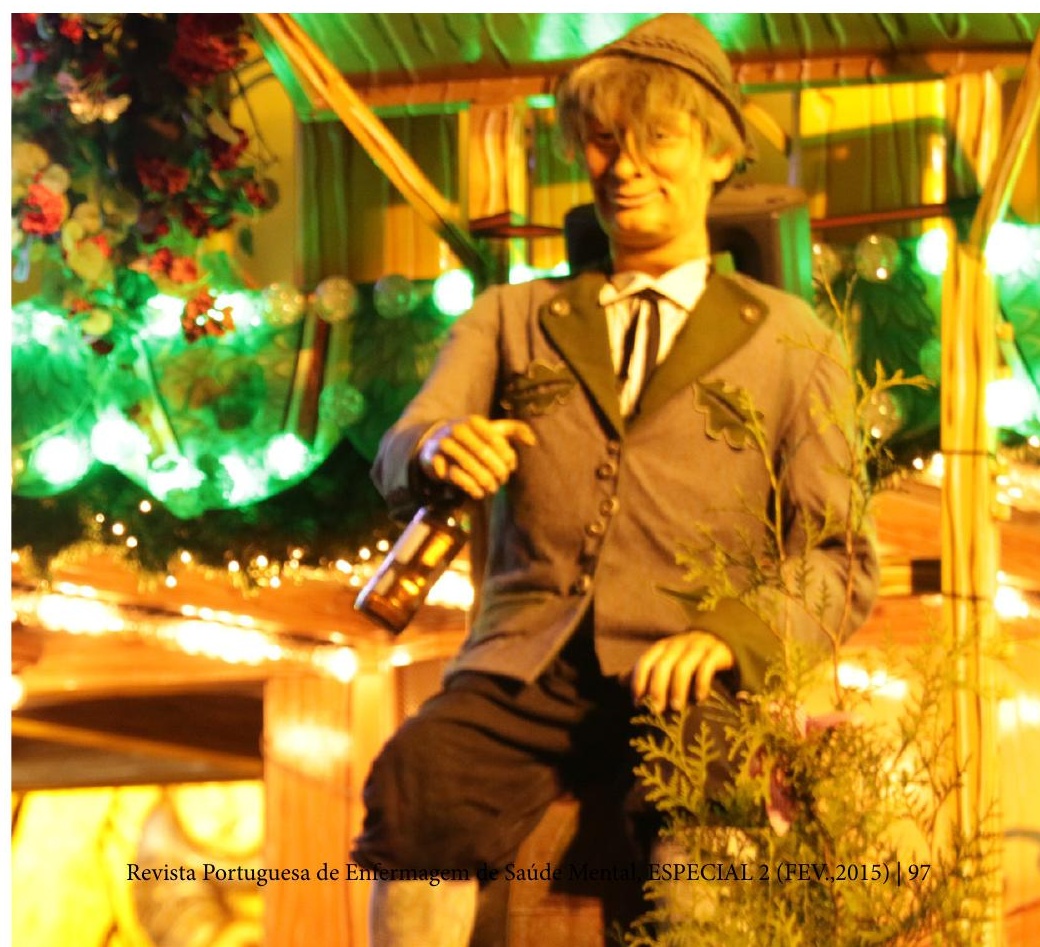


Tabela 2 - Distribuição dos participantes por Zonas do teste AUDIT de acordo com sexo, faixa etária, escolaridade e convívio com crianças. Belo Horizonte, 2013 ( $n=47)$

\begin{tabular}{|c|c|c|c|c|c|}
\hline $\begin{array}{l}\text { Zonas de } \\
\text { classificação }\end{array}$ & & $\begin{array}{l}\text { Zona I } \\
(0-7 \\
\text { pontos }) \\
\%\end{array}$ & $\begin{array}{l}\text { Zona II } \\
(8-15 \\
\text { pontos }) \\
\%\end{array}$ & $\begin{array}{l}\text { Zona III } \\
(16-19 \\
\text { pontos }) \\
\%\end{array}$ & $\begin{array}{l}\text { Zona IV } \\
(>20 \\
\text { pontos }) \\
\%\end{array}$ \\
\hline & TOTAL & 61,7 & 6,4 & 14,9 & 17,0 \\
\hline Sexo & $\begin{array}{l}\text { Feminino } \\
\text { Masculino }\end{array}$ & $\begin{array}{l}91,6 \\
30,4\end{array}$ & $\begin{array}{l}0,0 \\
13,1\end{array}$ & & \\
\hline Faixa etária & $\begin{array}{l}18-30 \text { anos } \\
31-50 \text { anos } \\
51 \text { anos ou } \\
\text { mais }\end{array}$ & $\begin{array}{l}71,4 \\
52,9 \\
65,2\end{array}$ & & & \\
\hline Escolaridade & $\begin{array}{l}1^{\circ} \mathrm{Grau} \\
\text { Incompleto } \\
1^{\circ} \mathrm{Grau} \\
\text { Completo } \\
2^{\circ} \mathrm{Grau} \\
\text { Incompleto } \\
2^{\circ} \text { Grau } \\
\text { Completo }\end{array}$ & $\begin{array}{l}59,0 \\
80,0 \\
100,0 \\
50,0\end{array}$ & & & \\
\hline $\begin{array}{l}\text { Pessoas que } \\
\text { convivem } \\
\text { com crianças } \\
\text { e adolescentes }\end{array}$ & $\begin{array}{l}\text { Sim } \\
\text { Não }\end{array}$ & $\begin{array}{l}70,9 \\
43,7\end{array}$ & & & \\
\hline
\end{tabular}

Fonte: Dados da pesquisa: Alcoolismo em região de risco e vulnerabilidade social: diagnóstico e intervenção, 2013.

Ao final da aplicação, os pesquisadores informaram aos participantes os resultados do teste orientando-os sobre a classificação no AUDIT e fizeram abordagens educativas por meio de intervenções.

O Quadro 2 demonstra orientações que auxiliaram os pesquisadores no direcionamento de intervenções. Os pesquisadores executaram as intervenções considerando as zonas de classificação e as especificidades apresentadas por cada participante.

Quadro 2 - Zonas do AUDIT e suas respectivas intervenções

\begin{tabular}{|l|l|}
\hline Zonas do AUDIT & Intervenções \\
\hline Zona I & $\begin{array}{l}\text { Ações de educação em saúde, para ma- } \\
\text { nutenção do uso atual de álcool. }\end{array}$ \\
\hline Zona II & $\begin{array}{l}\text { Orientação básica sobre o uso de baixo } \\
\text { risco e sobre os possíveis riscos orgânic- } \\
\text { os, psicológicos ou sociais, que o usuário } \\
\text { pode apresentar se mantiver esse padrão } \\
\text { de consumo. }\end{array}$ \\
\hline Zona III & $\begin{array}{l}\text { Utilização da técnica de Intervenção } \\
\text { Breve e o monitoramento. }\end{array}$ \\
\hline Zona IV & $\begin{array}{l}\text { Motivar o usuário a procurar atendi- } \\
\text { mento especializado para acompan- } \\
\text { hamento do caso e encaminhar para o } \\
\text { serviço adequado. }\end{array}$ \\
\hline
\end{tabular}

Fonte: Adaptado de Ministério da Justiça (2014).
Dos adultos que convivem com crianças e adolescentes, 25,9\% situaram-se nas zonas III e IV. A convivência de crianças e adolescentes com usuários de álcool, tanto nas famílias quanto na comunidade, requer intervenção. Diante dessa realidade criou-se a ação de extensão Oficina de Arte, Saúde e Paz. Direcionada a pré-escolares de uma creche da comunidade, a ação abordou aspectos relacionados à educação em saúde com foco na prevenção ao uso de álcool e outras drogas, promoção de saúde e cultura de paz.

Foram realizadas oficinas lúdico-pedagógicas de contação de histórias e o uso de desenhos, fantoches, músicas, brincadeiras e rodas de conversas. Os temas trabalhados foram escolhidos levando em consideração os resultados evidenciados pela pesquisa, a vulnerabilidade das crianças para o consumo de substâncias psicoativas, bem como as demandas apresentadas pelas educadoras da creche.

O trabalho preventivo desenvolvido na comunidade tornou-se relevante pois, possibilitou a troca de saberes entre os envolvidos nas atividades, além de ter incentivado a realização de estratégias de prevenção ao uso de drogas, especialmente do álcool.

\section{DISCUSSÃO}

A partir dos resultados apresentados, verificou-se que a maioria dos entrevistados foi classificada na zona I, ou seja, faziam uso de baixo risco ou eram abstêmios. As intervenções em saúde para estes indivíduos visaram orientá-los quanto à importância de manter-se como consumidores de baixo risco, tendo em vista os malefícios do uso de álcool em grande escala. Além disso, é indispensável que esses indivíduos sejam incentivados a divulgar as vantagens do consumo de baixo risco, valendo-se desses como agentes precursores de boas práticas de saúde, tanto na família como na comunidade (Ministério da Justiça, 2014).

Observou-se também na zona I o maior número de mulheres, o que indica que estas fazem uso de baixo risco. Esse achado também é evidenciado por dados apresentados por Laranjeira et al. (2007), que aponta o uso de álcool pelas mulheres como de menor frequência e quantidade.

As orientações fornecidas aos indivíduos na zona II objetivaram a redução do uso de álcool e o esclarecimento sobre os riscos à saúde, potencializados por esse padrão de consumo. Para Jomar et al. (2012) são muitos os fatores contribuintes para o desenvolvimento de problemas relacionados ao álcool. 
Entre esses, a falta de conhecimento sobre os limites do uso do álcool e sobre os riscos associados ao seu uso excessivo, as influências sociais e ambientais, assim como hábitos e atitudes que favorecem o beber excessivo.

Com os classificados na zona III utilizou-se a técnica de Intervenção Breve, que consiste em, de maneira empática e por meio de uma escuta reflexiva, avaliar o consumo de álcool e os problemas relacionados a ele e encorajar o indivíduo a eleger fatores pessoais que o auxiliem na redução do consumo de álcool ou abstinência.

Deve-se realizar também, com esses usuários, o monitoramento, que oportuniza momentos de trocas entre participantes e equipe de saúde, a fim de verificar as metas que já foram alcançadas e aquelas que ainda precisam ser aperfeiçoadas (Ministério da Justiça, 2014). Durante a pesquisa, esse monitoramento foi realizado em encontros de terapia comunitária que ocorriam na comunidade, direcionados pelos profissionais da Atenção Básica. Esses profissionais ofereceram apoio para o enfrentamento dos problemas relacionados ao uso do álcool.

Dos classificados como padrão de uso nocivo e chance de diagnóstico de dependência de álcool, o sexo masculino está em maior número quando comparado ao sexo feminino. Esses resultados corroboram com Levantamento realizado por Laranjeira et al. (2007), o qual demonstrou que de cada quatro pessoas do sexo masculino que fazem uso de álcool na vida, no Brasil, uma se tornará dependente. Para o sexo feminino, essa proporção é dez para uma.

$\mathrm{Na}$ zona IV foram incluídos aqueles que possuem aumento na possibilidade do diagnóstico de dependência. Segundo Jomar et al. (2012), o AUDIT não diagnostica dependência. Sendo assim, as pessoas classificadas nas zonas IV precisaram de uma avaliação adicional sobre a dependência alcoólica.

Desse modo, foi necessário encaminhar os usuários classificados na zona IV a serviços de saúde. Os serviços indicados abrangem desde a consulta clínica com o médico da equipe de saúde da Atenção Básica até serviços especializados para alcoolistas existentes na área de abrangência da comunidade.

Considerando o convívio de adultos consumidores de álcool com crianças e adolescentes, Schenker e Minayo (2005), apontam que o consumo de bebidas alcoólicas pelos pais ou responsáveis faz com que o público infanto-juvenil crie uma ideia de permissão e naturalidade.
Considera-se que uma fragilidade dos resultados deste estudo, dada a impossibilidade dos pesquisadores, foi o não acompanhamento dos participantes classificados na zona IV, depois de encaminhados para a equipe da Atenção Básica ou serviços especializados para alcoolistas. Sugere-se que outros estudos dessa natureza incluam esse acompanhamento.

\section{CONCLUSÃO}

A realização desta pesquisa evidenciou o desconhecimento dos participantes em relação ao próprio padrão de consumo de álcool e consequências que o abuso de álcool pode acarretar na vida pessoal e social do indivíduo. Notou-se também que entre os participantes da pesquisa, havia pouco conhecimento das informações quanto às possibilidades de prevenção e tratamento dos problemas relacionados ao uso de álcool.

Ressalta-se a importância da manutenção de atividades de promoção à saúde e prevenção ao uso e abuso de álcool como parte do cuidado de saúde prestado pela equipe que assiste a população estudada.

\section{IMPLICAÇÕES PARA A PRÁTICA CLÍNICA}

O AUDIT é um instrumento utilizado na identificação dos problemas relacionados ao álcool visto que sua aplicação possibilita detectar problemas associados a diferentes padrões de uso do álcool. Permite também fornecer orientações, intervenções, e quando preciso direcionamentos a serviços de saúde especializados.

A utilização do AUDIT nos serviços de Atenção Básica, configura-se como um instrumento para a realização de ações de educação em saúde, podendo ser utilizado por toda a equipe de saúde. A prática de enfermeiros pode ser potencializada com a aplicação de instrumentos dessa natureza, realização de atividades educativas de promoção de saúde, bem como, ações no âmbito da prevenção do uso de álcool e outras drogas, especialmente, em comunidades socialmente vulneráveis como a deste estudo.

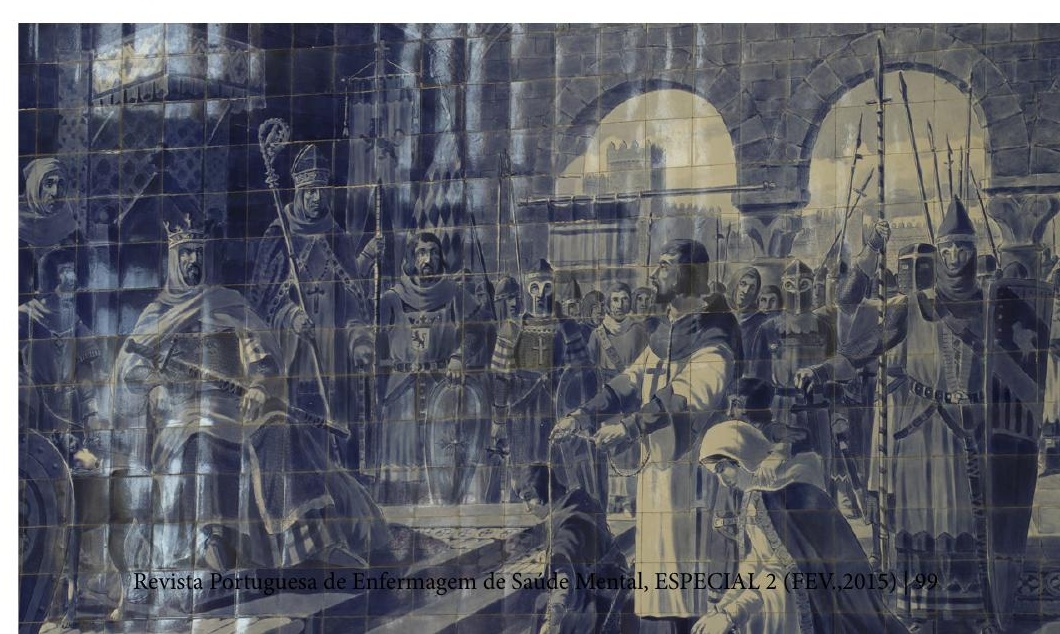




\section{REFERÊNCIAS BIBLIOGRÁFICAS}

Centro Brasileiro de Informações Sobre Drogas Psicotrópicas (2005). II levantamento domiciliar sobre o uso de drogas psicotrópicas no Brasil: Estudo envolvendo as 108 maiores cidades do país. São Paulo: Brasil.

Jomar, R. T., Paixão, L. A. R., e Abreu, A. M. M. (2012). Alcohol use disorders identification test (AUDIT) e sua aplicabilidade na atenção primária a saúde. Revista de Atenção Primária a Saúde, 15(1), 113-117. Acedido em http://aps.uff.emnuvens.com.br/aps/article/ view/1467/598

Laranjeira, R., Madruga, C. S., Ribeiro, M., Pinsky, I., Caetano, R., e Mitsuhiro, S. S. (2013). II levantamento nacional de álcool e drogas. São Paulo: Instituto Nacional de Ciência e Tecnologia para Políticas Públicas do Álcool e Outras Drogas. Acedido em http://inpad. org.br/wp-content/uploads/2013/04/LENAD_PressRelease_Alcohol_RVW.pdf

Laranjeira, R., Pinsky, I., Zaleski, M., e Caetano, R. (2007). I levantamento nacional sobre os padrões de consumo de álcool na população brasileira. Brasília: Secretaria Nacional de Políticas sobre Drogas.

Magnabosco, M. B., Formigoni, M. L. O. S., e Ronzani, T. M. (2007). Avaliação dos padrões de uso de álcool em usuários de serviços de atenção primária à saúde de Juiz de Fora e Rio Pomba (MG). Revista Brasileira de Epidemiologia, 10(4), 637-647. Acedido em http://www. scielo.br/pdf/rbepid/v10n4/20.pdf
Meloni, J. N., e Laranjeira, R. (2004). Custo social e de saúde do consumo do álcool. Revista Brasileira de Psiquiatria, 26(1), 7-10. Acedido em http://www.scielo. $\mathrm{br} / \mathrm{pdf} / \mathrm{rbp} / \mathrm{v} 26 \mathrm{~s} 1 / \mathrm{a} 03 \mathrm{v} 26 \mathrm{~s} 1 . \mathrm{pdf}$

Ministério da Justiça (2013). Prevenção do uso de drogas: Capacitação para conselheiros e lideranças comunitárias. Brasília: Secretaria Nacional de Políticas sobre Drogas.

Ministério da Justiça (2014). Sistema para detecção do uso abusivo e dependência de substâncias psicoativas: Encaminhamento, intervenção breve, reinserção social e acompanhamento (5a ed.). Brasília: Secretaria Nacional de Políticas sobre Drogas.

Minto, E. C., Corradi-Webste, C. M., Gorayeb, R., Laprega, M. R., e Furtado, E. F. (2007). Intervenções breves para o uso abusivo de álcool em atenção primária. Epidemiologia e Serviços de Saúde, 16(3), 207-220. Acedido em http://scielo.iec.pa.gov.br/pdf/ess/v16n3/ v16n3a07.pdf

Schenker, M., e Minayo, M. C. S. (2005). Fatores de risco e de proteção para o uso de drogas na adolescência. Ciência \& Saúde Coletiva, 10(3), 707-717. Acedido em www.scielosp.org/pdf/csc/v10n3/a27v10n3.pdf

Tripp, D. (2005). Pesquisa-ação: Uma introdução metodológica. Educação e Pesquisa, 31(3), 443-466. Acedido em http://www.scielo.br/pdf/ep/v31n3/ a09v31n 3 